\title{
Radiative cooling of shocked gas in stellar atmospheres
}

\section{Self-consistent LTE shock wake model with Fe lines and $\mathrm{H}$ continua contributions}

\author{
A. B. Fokin ${ }^{1,4}$, G. Massacrier ${ }^{2}$, and D. Gillet ${ }^{3}$ \\ ${ }^{1}$ Institute of Astronomy of the Russian Academy of Sciences, 48 Pjatnitskaya Str., Moscow 109017, Russia \\ 2 CRAL, UMR 5574 du CNRS, École Normale Supérieure de Lyon, 46 allée d'Italie, 69364 Lyon Cedex 07, France \\ 3 Observatoire de Haute Provence, CNRS, 04870 Saint Michel l'Observatoire, France \\ ${ }^{4}$ Isaak Newton Institute Moscow Branch
}

Received 28 March 2003 / Accepted 13 January 2004

\begin{abstract}
The hot radiative shock wake structure, including the radiative cooling due to $\mathrm{H}$ continuum and the $\mathrm{Fe}$ lines under typical conditions of stellar atmospheres, is considered. The proposed iterative method of calculation is stable and gives the temperature profile with a good precision (2-5\%). The dependence of the wake structure on the preshock parameters is studied, and the line cooling is compared to that of continua in a more self-consistent way. We confirm that the iron cooling is very efficient for low preshock densities (less or equal to $10^{-11} \mathrm{~g} / \mathrm{cm}^{3}$ for Population I, and less or equal to $10^{-12} \mathrm{~g} / \mathrm{cm}^{3}$ for Population II). However, for higher densities, $10^{-10} \mathrm{~g} / \mathrm{cm}^{3}$ and more, especially for the Population II composition, the line cooling becomes secondary with respect to the continuum. As a consequence, the iron cooling is expected to be efficient for pulsating stars having low $R / M$ ratios, like classical Cepheids or RR Lyrae, and much less efficient for W Vir, RV Tauri and Miras. This is discussed in view of the well-known drastic difference in the observed emission features between these two stellar groups.
\end{abstract}

Key words. shock waves - stars: oscillations - stars: atmospheres

\section{Introduction}

In Paper I (Fokin et al. 2000, FMG1 hereafter) we considered a restricted simplified problem of the line radiative transfer across the radiative shock wake. We assumed to be known the temperature distribution in the wake and neglected the variation of the gas velocity with the distance from the shock front (with a jump at the front). Under the LTE approximation, we studied the radiative cooling and heating zones provoked by one line, and roughly estimated the total contribution from the Fe lines to the cooling in the wake. This contribution was found to be important with respect to the contribution from hydrogen continua.

However, as mentioned in FMG1, such an approximate analysis cannot give us information about the thermal structure of the shock wake, which is of a great importance. For this, the direct detailed calculation of the energy losses of the gas is needed.

In this second paper we present the results of such detailed calculations of the stationary shock wake structure in typical conditions of stellar atmospheres $(\rho=2 \times$ $\left.10^{-13}-2 \times 10^{-10} \mathrm{~g} \mathrm{~cm}^{-3}\right)$. The unperturbed temperature is varied from $3000 \mathrm{~K}$ to $7000 \mathrm{~K}$. As in FMG1, we consider only

Send offprint requests to: D. Gillet, e-mail: gillet@obs-hp. fr radiative cooling and ignore the cooling/heating due to the adiabatic processes in the wake. We included lines of Fe I and Fe II, and the $\mathrm{H}$ and $\mathrm{H}^{-}$continua. For the line transfer analysis, we used a more sophisticated numerical code than in FMG1. However, we kept the Dopplerian profile for the line absorption, and the LTE atomic populations of all species. To estimate the free electron concentration, we used the Saha equation for ionizations of $\mathrm{H}, \mathrm{He}$ and the first three ionizations of the 21 most important heavier elements. The calculations are performed for Population I and II compositions.

In the next section we describe our shock model and physical constraints of the problem. In Sect. 3 we outline the numerical method of the line transfer calculation and the iterative technique to find the temperature distribution in the wake. Sections 4 and 5 contain the results and their applications to the atmospheres of pulsating stars. Finally, Sect. 6 contains the general discussion of the obtained results.

\section{Physical assumptions of the shock model}

As in FMG1, we consider here a stationary shock, with all physical quantities depending only on the distance from the front.

The hypothesis of stationarity of the shock wave implies that the preshock gas parameters (mainly the density) vary 
much more slowly than the time required to achieve equilibrium in the whole wake. Normally, the gas velocity just behind the front is of the order of several kilometers per second and decreases with the distance from the front. Provided that the characteristic space scale of the wake is about $10^{7} \mathrm{~cm}$, this approximately gives the time of passage of an element of gas through the wake, $\tau_{\text {pass }} \approx 50 \mathrm{~s}$, which represents approximately the relaxation time of the wake. The time in which a shock crosses the atmosphere depends on the stellar type. In "compact" atmospheres, such as RR Lyrae, this time is about $1500 \mathrm{~s}$, or about 30 times the $\tau_{\text {pass }}$. In atmospheres of RV Tauri stars this time is much longer - about $10^{6} \mathrm{~s}$. On the other hand, the gas density varies (decreases) very rapidly towards the surface in RR Lyrae stars, while it does so much slowly in RV Tauri or Mira stars (due to low gravity). This suggests that the stationarity hypothesis should be more plausible for the cooler giants than for hot stars with high gravity.

In contrast to the shock model used in FMG1, in the present study we use a more physically plausible and less constrained model. Firstly, we reject the assumptions of constant mass density $\rho$ and velocity $v$ in the wake, and use mass and momentum conservation equations to determine $\rho$ and $v$.

Secondly, we use here the radiative transfer solution method that is valid for spherically-symmetric atmospheres. However, in this paper we consider a shock model that is very close to the plan-parallel limit. For this, we fixed the shock front radius at $100 R_{\odot}$, which is by $4-5$ orders of magnitude larger than typical wake widths. We choose the inner boundary of our model at the level where the optical depth in the continuum near the lines is of the order of 10 .

Also, the opacity coefficients in the line and in the nearby continuum are calculated from the populations of the corresponding atomic levels, evaluated in turn from the solution of the LTE equations of state.

The LTE assumption is a simplification that is usually adopted because of the well-known technical difficulties of the full non-LTE calculations. It is clear that it may be not very correct when applied to the cooling rate calculations. However, recently Woitke \& Sedlmayr (1999) performed calculations of the iron cooling in stellar winds using the Sobolev method and presented a comparison of the LTE/non-LTE results. According to the authors, the LTE and full non-LTE solutions are approximately alike as $\log n_{\mathrm{H}} \geq 12.5\left(\rho \geq 5 \times 10^{-12} \mathrm{~g} / \mathrm{cm}^{3}\right)$ both for Fe I and Fe II. This condition corresponds to our shock model, which allows us to suggest that the LTE assumption used here is acceptable.

Our shock model is represented schematically in Fig. 1. The fine structure of the shock wave has already been presented in FMG1 (see Fadeyev \& Gillet 2000, 2001).

Our main hypothesis is that the precursor, the viscous front and the region of the temperature relaxation (i.e. the whole zone "c-d" in Fig. 1) are transparent in all iron spectral lines (and in their nearby continua). The optical width of the preshock zone, i.e. of the unperturbed atmosphere before the viscous front, is not critical for the line cooling rate in the wake, as in FMG1 we show that the cooling rate does not depend on the optical depth of the viscous front, where the Doppler jump occurs. This hypothesis cannot be proved strictly due to

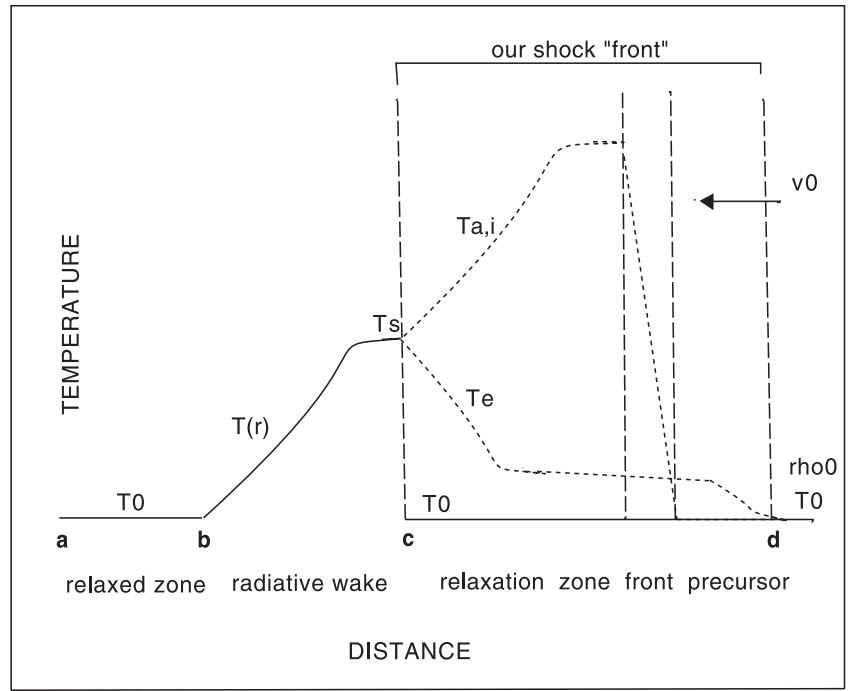

Fig. 1. Schematic diagram representing the fine structure of the shock wave. The model of the radiative shock wake, adopted in this study, is shown by the solid lines. The four input parameters of the problem are: $v_{0}, \rho_{0}, T_{0}$ (the velocity, mass density and temperature of the unperturbed preshock gas) and $T_{\mathrm{s}}$, the temperature at the outer boundary of our wake.

obvious technical difficulties, but there are several arguments favoring the fact that, at least in most iron lines, this region is transparent. Since in FMG1 we demonstrated that the cooling in the wake is controlled mainly by the number of lines, provided that their strength is not very small, we believe that even if there is a (supposedly small) number of lines that are strong enough to be opaque in the zone "c-d", they cannot affect seriously the total cooling rate controlled by numerous fainter iron lines. Practically, in this study we included the iron lines with wavelength $\lambda$ between $1000 \AA$ and $20000 \AA$, excluding those falling in the Lyman continuum.

Another hypothesis is that the preshock and the far postshock regions have a constant temperature $T=T_{0}$. This reflects the situation in stellar atmospheres, which are almost isothermic off the shocked region (but not strictly).

In the following, we do not consider at all the structure of the "preshock" zone (to the right of "c") assuming in this zone the same $\rho_{0}, v_{0}$ and $T_{0}$ as in the true unperturbed region far ahead the shock. Our goal is to estimate the temperature in the wake as a function of the distance from the front. Thus, our wake is represented by the zone " $\mathrm{a}-\mathrm{c}$ " in the figure, and our "preshock" has the same parameters as the region above the point " $\mathrm{d}$ ". The problem of the radiative cooling is now controlled only by 4 free parameters, except for the chemical composition and the position of the shock within the atmosphere: 3 characteristics of the preshock gas $-\rho_{0}, v_{0}$ and $T_{0}$ (which we shall apply for the whole region "c-d") and the temperature $T_{\mathrm{s}}$ at the point "c". The function $T(r)$ is found from the detailed calculation of the radiative transfer in all lines and continua, and subsequent evaluation of the radiative cooling rate. The density is obtained from the relevant Rankine-Hugoniot relations. 
We summarize then the basic assumptions of our model as follows:

- the shock is stationary;

- here we choose the shock wake model close to a planparallel case by putting $R_{\text {shock }}=100 R_{\odot}$. The gas velocity and density in the wake are calculated using conservation laws for mass and momentum with the thermal radiative pressure included;

- the cooling is supposed purely radiative (see next section);

- the fine shock structure before the radiative wake is supposed transparent in the iron lines and $\mathrm{H}$ continua, except for the Lyman continuum;

- the cooling rate due to continuum is calculated under LTE including the $\mathrm{H}$ and $\mathrm{H}^{-}$bound-free and free-free continua only. The Lyman continuum is ignored;

- the iron line cooling rates are calculated under LTE including only Fe I and Fe II lines. The line absorption profile is supposed Dopplerian calculated using the constant microturbulent velocity of $1 \mathrm{~km} \mathrm{~s}^{-1}$;

- the parameters of the preshock region (i.e. $\rho_{0}, v_{0}$ and $T_{0}$ ) are supposed constant. The structure of this region is not considered.

\section{Basic equations and numerical method}

\subsection{Gas-dynamic equations}

The general energy equation of the gas in the presence of the radiation field, formulated in the co-moving frame, is the second law of the thermodynamics and can be written as (see e.g., Mihalas 1978)

$$
\frac{\mathrm{D} e_{\mathrm{g}}}{\mathrm{D} t}+P_{\mathrm{g}} \frac{\mathrm{D}(1 / \rho)}{\mathrm{D} t}=\frac{4 \pi}{\rho} \int_{0}^{\infty}\left(\chi_{v} J_{v}-\eta_{v}\right) \mathrm{d} v,
$$

where $e_{\mathrm{g}}$ is the gas internal energy per gram, $P_{\mathrm{g}}=\rho R T / \mu$ is the gas pressure, $\mu$ is the mean molecular weight, $\rho$ the mass density, $\chi_{v}$ and $\eta_{v}$ the monochromatic opacity and emissivity, and $J_{v}$ the mean intensity in the comoving frame. The right hand side of Eq. (1) is the total radiative cooling rate $Q$ (in $\mathrm{erg} / \mathrm{g} / \mathrm{s}$ ). $e_{\mathrm{g}}$ includes the gas-kinetic (thermal) energy as well as the ionization/excitation energies of ions. In the temperaturedensity range of interest for this paper, adding the ionization energy of $\mathrm{H}$ to the thermal energy would in practice be sufficient.

In a stationary plan-parallel case the adiabatic term in the left-hand side of the Eq. (1) is always negative and provides some gas heating behind the front, competing with the radiative cooling. By simple estimations and some test calculations one can show that in a typical case of our shock model the adiabatic term is about $10 \%$ or less of the radiative one. The reason is that the gas loses energy mainly at the expense of the ionization/excitation pool. We neglect the adiabatic heating in this paper, but it should probably be retained in other conditions or for spherical moving flows.

The Lagrangean time derivative is $\frac{\mathrm{D}}{\mathrm{D} t}=\frac{\partial}{\partial t}+v \frac{\partial}{\partial r}$, where $v$ is the local velocity in the shock front frame (note that $v<0$ ).
For a stationary shock wave and ignoring the adiabatic term, Eq. (1) becomes

$$
\frac{\mathrm{d} e_{\mathrm{g}}}{\mathrm{d} r}=\frac{4 \pi}{\rho v} \int_{0}^{\infty}\left(\chi_{v} J_{v}-\eta_{v}\right) \mathrm{d} v .
$$

In LTE and for given chemical abundances the gas energy $e_{\mathrm{g}}$ is given as a function of the temperature and the density. Thus

$\frac{\mathrm{d} e_{\mathrm{g}}}{\mathrm{d} r}=\left(\frac{\partial e_{\mathrm{g}}}{\partial T}\right)_{\rho} \frac{\mathrm{d} T}{\mathrm{~d} r}+\left(\frac{\partial e_{\mathrm{g}}}{\partial \rho}\right)_{T} \frac{\mathrm{d} \rho}{\mathrm{d} r}$.

To determine both $T$ and $\rho$ as well as the velocity $v$ Eq. (2) is supplemented with the conservation laws for mass and momentum. For a stationary plane-parallel flow and neglecting viscosity and external forces this results in equations similar to the two first Rankine-Hugoniot relations:

$\rho v=\rho_{0} v_{0}$,

$P_{\mathrm{g}}+P_{\mathrm{rad}}+\rho v^{2}=P_{\mathrm{g}, 0}+P_{\mathrm{rad}, 0}+\rho_{0} v_{0}^{2}$,

where subscript 0 denotes the value of a quantity in the preshock region, $P_{\text {rad }}=a \cdot T^{4} / 3$ is the thermal radiative pressure and $a$ the radiative constant.

Here we introduce several simplifications. We omitted the spherical factor $4 \pi r^{2}$ because the characteristic width of the wake $\Delta r_{w} \approx 10^{7} \mathrm{~cm}$ is much smaller than the radii of pulsating stars. For example, for the compact RR Lyrae stars $\Delta r_{w} / R_{\mathrm{RR}} \approx 2 \times 10^{-5}$, while for RV Tauri stars it is less than $10^{-6}$. Next, in Eq. (5) we neglected the gas viscosity. After this, the above relations allow to express $\rho$ and $v$ as a function of $T$, so that Eq. (2) together with Eq. (3) can give $T(r)$ once the radiative transfer problem is solved.

We found, however, that the inclusion of the radiative pressure has only a small effect on the density structure of the wake, and has a negligible effect on the temperature structure. This is explained by the fact that the radiative pressure is maximal in the hottest zone of the wake, which is extremely short. The energy losses in this zone are small, so the T-profile in the wake remains almost unaffected.

Below we give a preliminary simplified analysis of the shock cooling ignoring the radiative pressure. Figure 2 illustrates for six different typical preshock conditions the relation between the temperature and the density, which obtains from eliminating $v$ from Eqs. (4) and (5):

$$
\frac{R T}{\mu}=\frac{\rho_{0} v_{0}^{2}}{\rho}\left(1+\frac{1}{M_{0}^{2}}-\frac{\rho_{0}}{\rho}\right) .
$$

Here $M_{0}=\left|v_{0}\right| / \sqrt{\left(R T_{0} / \mu_{0}\right)}$ is the preshock Mach number. The part of each curve covered by the radiative wake is on the decreasing side from $T_{\mathrm{s}} \simeq 10000 \mathrm{~K}$ (see below) to the relaxed temperature $T_{0}$ (here $5000 \mathrm{~K}$ ). A wealth of information can be inferred from such plots.

It is seen that the radiative wake crosses the ionization strip of hydrogen. The main variations of the gas energy density $e_{\mathrm{g}}$ are actually due to the recombination of $\mathrm{H}$, except in the far wake (low temperature) or for high postshock densities where hydrogen is mainly neutral and thermal energy dominates. Note 


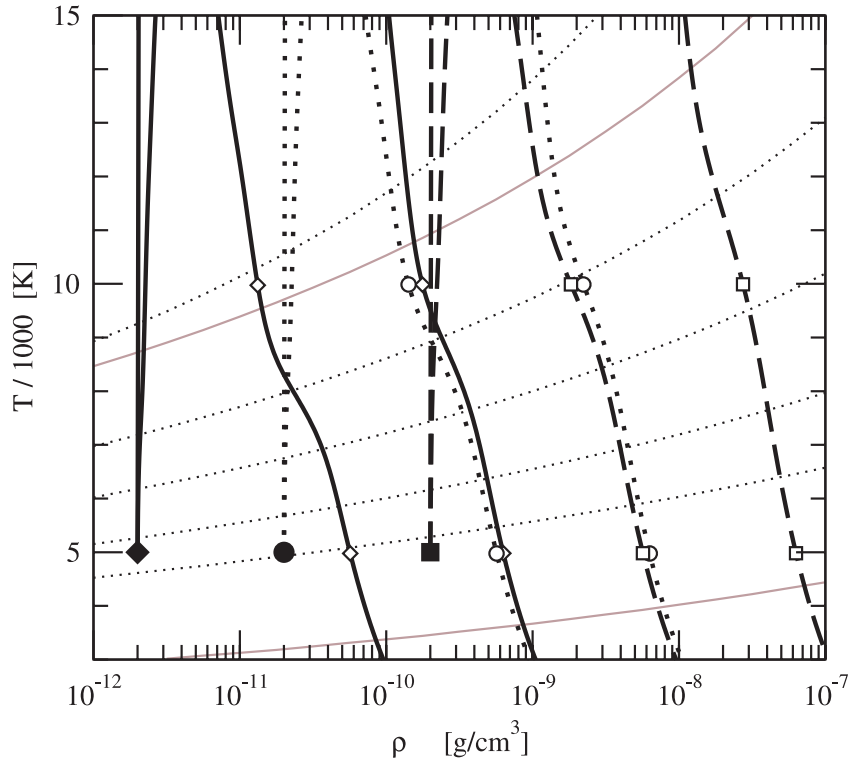

Fig. 2. Shock traces (heavy lines) in the $(\rho, T)$ plane as resulting from Hugoniot relations (for solar abundances), for initial preshock conditions $T_{0}=5000 \mathrm{~K}$ and $\rho_{0}=2 \times 10^{-12}$ (solid), $2 \times 10^{-11}$ (dotted) and $2 \times 10^{-10} \mathrm{~g} \mathrm{~cm}^{-3}$ (dashed). For each of these conditions two initial velocities are considered: $v_{0}=30$ and $100 \mathrm{~km} \mathrm{~s}^{-1}$, leading to two curves with the rightmost associated to the highest velocity. Initial points are marked by solid symbols, empty ones mark the limits of the radiative wake. Dotted (light) lines are contour plots for the fractional abundance of ionized hydrogen, with this fraction value equal to $0.1 \%, 1 \%$, $10 \%, 50 \%$ and $99 \%$ from bottom to top. On the lowest (resp. highest) solid gray line, Fe I and Fe II (resp. Fe II and Fe III) have equal densities. Fe II is dominant inbetween.

that starting from a lower ionization state dense wakes must radiate a little less energy (per gram). Continuum opacities due to hydrogen are also expected to show strong variations in going from the point just behind the shock front to the far tail. On most part of the wake Fe II is the main ion stage for iron. Its contribution to the line cooling rate actually dominates the Fe I one. Note that for shocks on the left in Fig. 2 (e.g. $\left.\rho_{0}=2 \times 10^{-12} \mathrm{~g} \mathrm{~cm}^{-3},\left|v_{0}\right|=30 \mathrm{~km} \mathrm{~s}^{-1}\right)$ and just behind the shock front, the Fe III population is not negligible. It could increase the cooling rate but its contribution is not included in this paper.

For the shocks we study $M_{0} \gg 1$. As in the wake $\rho \gg \rho_{0}$, Eqs. (6) and (4) simplify into

$$
\frac{R T}{\mu} \simeq \frac{\rho_{0} v_{0}^{2}}{\rho}, \quad v=\frac{\rho_{0} v_{0}}{\rho} \simeq \frac{R T / \mu}{v_{0}} .
$$

Shocks with identical value of incident kinetic energy $\rho_{0} v_{0}^{2}$ describe the same $(\rho-T)$-path in their wake as can be seen in Fig. 2 for the couples $\left(\rho_{0}, 100 \mathrm{~km} \mathrm{~s}^{-1}\right)$ and $\left(10 \times \rho_{0}, 30 \mathrm{~km} \mathrm{~s}^{-1}\right)$. However this path will be covered with different speeds. From previous relations Eq. (2) can be formally written

$A(T) \frac{\mathrm{d} T}{\mathrm{~d} r}=v_{0} \frac{4 \pi}{\rho} \int_{0}^{\infty}\left(\chi_{v} J_{v}-\eta_{v}\right) \mathrm{d} v \equiv v_{0} Q$,

where $A$ is some known function of $T$ and of the parameter $\rho_{0} v_{0}^{2}$. The cooling rate $Q$ results from the solution of the (non local) transfer equation. If we suppose however that it can be determined from local conditions (i.e. $Q \equiv Q(T, \rho)$ ), then Eq. (8) implies that for shocks with given $\rho_{0} v_{0}^{2}$ the wake width scales as $1 / v_{0}$ : the higher the initial velocity $v_{0}$, the narrower the wake width. This is easily understood from Eq. (7): the velocity $v$ in the wake being smaller, more time is taken by a gas parcel to cross a given distance, hence a greater cooling. The condition of "local" cooling rate is fulfilled whenever $J_{v} \ll \eta_{v} / \chi_{v}=B_{v}(T)$, at least for the main contributors to the cooling rate. Such a situation occurs for strong lines of iron behind the front as long as they are still thin. Our conclusion on the wake width is indeed verified in our numerical results down to, say, 7000-8000 K for not too dense shocks. The profile of the rest of the wake can only be determined from a simultaneous solution of the radiative transfer problem.

\subsection{Radiative transfer equation and atomic populations}

Below we discuss our transfer code which was created originally for a spherically-symmetric moving atmosphere. For the present shock model we adopted it for a plane-parallel case.

The co-moving transfer equation for the monochromatic specific intensity $I(r, \mu, v)$ in a spherically-symmetric moving atmosphere is (Castor 1972)

$$
\begin{aligned}
\mu \frac{\partial I_{v}}{\partial r}+\frac{1-\mu^{2}}{r} & \frac{\partial I_{v}}{\partial \mu}-\frac{v v}{c r}\left(1-\mu^{2}+\mu^{2} \frac{\mathrm{d} \ln v}{\mathrm{~d} \ln r}\right) \frac{\partial I_{v}}{\partial v} \\
& =\eta_{v}-\chi_{v} I_{v}
\end{aligned}
$$

where $r$ is the radius, $v$ the local frequency, $v$ the radial velocity, $\eta_{v}(r)$ and $\chi_{v}(r)$ the emission and absorption coefficients, respectively. In Eq. (9) the time derivative, the advection and aberration terms are neglected. This simplification does not affect the accuracy of the solution much, but it considerably simplifies the calculation. For this, we use the numerical method developped in Fokin (1992). Omitting the details, below we describe only the main aspects of this procedure.

We use the discrete-ordinate method of Hummer \& Rybicky (1971) (Fig. 3), in which the transfer equation is resolved along a series of parallel rays, from the base of the atmosphere (shown as a hashed sector) to the surface, and then the mean intensity at each grid point is obtained by integrating intensities over all rays.

Along each ray shown in the figure, the transfer equation in both directions can be expressed as

$\pm \frac{\partial I^{ \pm}(z, v)}{\partial z}-\frac{\partial I^{ \pm}(z, v)}{\partial v} \frac{\partial v}{\partial z}=\eta(z, v)-\chi(z, v) I^{ \pm}(z, v)$,

where $z$ is the distance along the ray, and indexes "+" and "-_" indicate the outward and inward direction of photons, respectively. The frequency derivative $\partial v / \partial z=v / c \partial(v \mu) / \partial z$ reflects the variation of the intensity frequency along the ray (i.e. along the path of a photon) due to the Doppler effect, seen from the co-moving frame. After discretization, Eq. (10) is solved on the grid $(z, v)$ using the Feautrier method.

The absorption and emission coefficients in the case of the complete redistribution are

$\chi_{v}=\chi_{L} \phi_{v}+\chi_{\text {cont }}(z, v)$ 


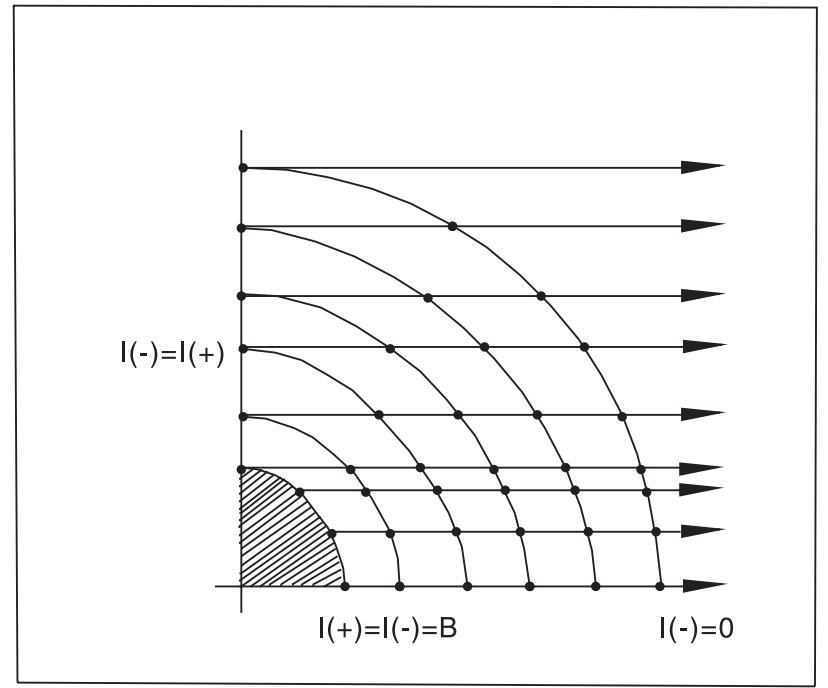

Fig. 3. The Hummer \& Rybicky scheme for the transfer equation solution in a spherical symmetry. The hashed sector denotes the subphotospheric optically thick inner region. The arrows indicate the direction towards the observer. The boundary conditions for $I^{-}$and $I^{+}$are also indicated.

and

$\eta_{v}=\eta_{L} \phi_{v}+\eta_{\mathrm{cont}}(z, v)$

where $\phi_{\nu}$ is the normalized line absorption profile and

$\chi_{L}=\left(\frac{\pi e^{2}}{m_{\mathrm{e}} c}\right) f_{l u}\left(n_{l}-\frac{g_{l}}{g_{u}} n_{u}\right)$

$\eta_{L}=\left(\frac{\pi e^{2}}{m_{\mathrm{e}} c}\right) f_{l u} \frac{g_{l}}{g_{u}} \frac{2 v_{l u}^{3} h}{c^{2}} n_{u}$,

with the oscillator strength related to the Einstein coefficient through

$f_{l u}=\left[\frac{8 \pi v_{l u}^{2}}{c^{2}}\left(\frac{\pi e^{2}}{m_{\mathrm{e}} c}\right) \frac{g_{l}}{g_{u}}\right]^{-1} A_{u l}$.

The notations are as usual (e.g., Mihalas 1978). For the line source function, $S_{v}(z)=\eta_{v}(z) / \chi_{v}(z)$, we adopt the two-level atom form

$S_{\nu}(z)=(1-\epsilon) \int_{0}^{\infty} \phi_{\nu} J_{\nu} \mathrm{d} v+\epsilon B_{v}(T)$.

With respect to the usual $\epsilon(v, z)$, depending for the nonLTE case on $v$ and on $z$, in Eq. (16) we assume $\epsilon$ constant within the whole atmosphere. In this, $\epsilon=1$ corresponds to the pure LTE case, while $\epsilon=0$ describes pure scattering. Such an approximation, though rough, is aimed at testing non-LTE effects on the line cooling. Except where indicated, we performed calculations with $\epsilon=1$ (LTE case). We note that because of such a simple form of $S$, we have no problem with convergency of the $\Lambda$-iterations (see Buchholz et al. 1994).
In the $\chi_{\text {cont }}(z, v)$, we include the $\mathrm{H}$ bound-free transitions for a 10 -level atom, $\mathrm{H}^{-}$bound-free and $\mathrm{H}$ free-free transitions. We use the following effective cross-sections (in $\mathrm{cm}^{2}$ ):

- for $\mathrm{H}_{\mathrm{bf}}$ :

$$
\begin{aligned}
& \sigma(v, n)=2.815 \times 10^{29} \frac{1}{n^{5}} \frac{1}{v^{3}}, \quad h v \geq \frac{13.6}{n^{2}} \mathrm{eV}, \\
& \sigma_{\mathrm{bf}}(v)=\sum_{n=1}^{10} \sigma(v, n) ;
\end{aligned}
$$

- for $\mathrm{H}_{\mathrm{ff}}$ :

$\sigma(v, T)=3.69 \times 10^{8} \frac{n_{\mathrm{e}}}{T^{1 / 2} v^{3}}(1-\exp (-h v / k T)) ;$

- for $\mathrm{H}_{\mathrm{bf}}^{-}$:

$\sigma=3.7 \times 10^{-17}$ if $2.55 \times 10^{14}<v<4.8 \times 10^{14} \mathrm{~Hz}$,

and $\sigma=2.7 \times 10^{-17}$ for other $v$.

The free-free transitions of $\mathrm{H}^{-}$are important only for $\lambda>$ $15000 \AA$.

In this paper we use the LTE populations, so the calculation of the $n_{i}$ and, consequently, $\chi_{v}$ is greatly simplified. We calculate the atomic populations of all elements using the Saha \& Boltzman relations. Consequently (see e.g., Mihalas 1978), for a given ionization state $j$, the ratio of the atomic population at levels $u$ and $l$ is

$\frac{n_{u j}}{n_{l j}}=\frac{g_{u j}}{g_{l j}} \mathrm{e}^{-h v_{u l} / k T}$.

For the total concentration of atoms in the ionization state $j$ we have

$N_{j}=\sum_{i} n_{i j}=\frac{n_{0 j}}{g_{0 j}} U_{j}(T)$,

where

$U_{j}(T)=\sum_{i} g_{i j} \mathrm{e}^{-\chi_{i j} / k T}$

is the so-called partition function and $\chi_{i j}$ is the excitation energy of the level $i j$. The values of $U_{j}(T)$ are tabulated for different elements in Irwin (1981). This also gives

$\frac{n_{i j}}{N_{j}}=\frac{g_{i j}}{U_{j}(T)} \mathrm{e}^{-\chi_{i j} / k T}$.

The ratio of the basic atomic populations in the two nearest ionization levels is

$\frac{n_{0 j}}{n_{0, j+1}}=\frac{n_{\mathrm{e}}}{2}\left(\frac{h^{2}}{2 \pi m k T}\right)^{3 / 2} \frac{g_{0 j}}{g_{0, j+1}} \mathrm{e}^{\chi_{I j} / k T}$,

where $\chi_{I j}$ is the ionization energy of the ion $j$. Applying the Boltzman equation, we get

$\frac{n_{i j}}{n_{0, j+1}}=C_{I} n_{\mathrm{e}} T^{-3 / 2} \frac{g_{i j}}{g_{0, j+1}} \mathrm{e}^{\left(\chi_{I j}-\chi_{i j}\right) / k T}$,

where $C_{I}=2.07 \times 10^{-16}[\mathrm{cgs}]$, or

$\frac{n_{i j}}{N_{j+1}}=n_{\mathrm{e}} \frac{C_{I} T^{-3 / 2}}{U_{j}(T)} \mathrm{e}^{\left(\chi_{I j}-\chi_{i j}\right) / k T}$, 
and

$\frac{N_{j}}{N_{j+1}}=n_{\mathrm{e}} \frac{U_{j}(T)}{U_{j+1}(T)} C_{I} T^{-3 / 2} \mathrm{e}^{\chi_{I j} / k T}$.

In the $n_{\mathrm{e}}$ calculation we use the same metallicity coefficient $\beta=$ $[\mathrm{Fe} / \mathrm{H}]$ (which is one of the input parameters) for all elements, so the helium mass fraction is $Y=1-X-0.02 \times 10^{\beta}$. In the following we take $\beta=0$ for Population $\mathrm{I}$, and $\beta=-1$ for Population II. Also, we take into account only the neutral state and two first ionizations. We include the contribution of the 23 most abundant elements: $\mathrm{H}, \mathrm{He}, \mathrm{C}, \mathrm{N}, \mathrm{O}, \mathrm{Ne}, \mathrm{Na}, \mathrm{Mg}$, Al, Si, P, S, Cl, Ar, K, Ca, Ti, Cr, Mn, Fe, Co, Ni, Ba. The third ionization of these species has very high ionization energy (about 30 to $80 \mathrm{eV}$ ), so this state can be ignored.

The above formalism allows us to find all needed populations, the line and continuum absorptions, the mean specific intensity and, finally, to evaluate the radiative cooling rate as function of the distance from the shock front.

Taking into account the relatively small width of the shock wake (see above), here we adapted this method to the planeparallel case.

\subsection{Cooling rates}

We begin with expressions for the line and continuum cooling rates. As we do not consider here the full atmospheric modelling and look for a self-consistent structure of the shock wake only, we should use somewhat modified expressions for these rates, which would take into account the radiative equilibrium of the atmosphere without shocks. Indeed, since in our simplified model we suppose that the temperature in the relaxed atmosphere is constant, $T_{0}$, we must put the cooling/heating rates to zero at $T<T_{0}$. For this purpose, our equations for $Q_{\text {line }}$ and $Q_{\text {cont }}$ contain a factor $B_{v}(T)-B_{v}\left(T_{0}\right)$.

Using the results of FMG1 and the above reasoning, we rewrite the cooling rate due to one $L$ th line

$$
Q_{\text {lines }, L} \equiv\left(\frac{\mathrm{D} e_{\mathrm{g}}}{\mathrm{D} t}\right)_{\text {lines }, L} \equiv \frac{4 \pi}{\rho} \chi_{L}\left(\frac{\overline{J_{L}}}{S_{L}}-1\right) S_{L}
$$

as

$$
\begin{aligned}
Q_{\text {lines }, L} & =\frac{4 \pi}{\rho} n_{l} f_{\text {osc }}\left(\frac{\pi e^{2}}{m_{\mathrm{e}} c}\right)\left(1-\frac{g_{l} n_{u}}{g_{u} n_{l}}\right) \\
& \cdot\left(\frac{\overline{J_{L}}}{S_{L}}-1\right)\left(B_{v}(T)-B_{v}\left(T_{0}\right)\right),
\end{aligned}
$$

where $n_{l}$ and $n_{u}$ are the atomic populations (in $\left[\mathrm{cm}^{-3}\right]$ ) of the lower and upper levels, respectively, $f_{\text {osc }}$ the oscillator strength, $g_{l}$ and $g_{u}$ the statistical weigths of the lower and upper atomic levels, $B_{v}(T)$ and $B_{v}\left(T_{0}\right)$ the Planck functions at the line frequency $v$ corresponding to the temperatures $T$ and $T_{0}$ and $\overline{J_{L}}$ the scattering integral.

The continuum cooling rate is

$Q_{\text {cont }} \equiv\left(\frac{\mathrm{D} e_{\mathrm{g}}}{\mathrm{D} t}\right)_{\text {cont }}=\frac{4 \pi}{\rho} \int_{0}^{\infty} \chi_{\text {cont }}(v)\left(J_{v}-S_{v}\right) \mathrm{d} v$,

where $\chi_{\text {cont }}(v)$ includes only pure absorption, and is discussed above. Practically, the evaluation of $Q_{\text {cont }}$ is more difficult, because in our model its evaluation cannot be physically correct. Even under LTE hypothesis, a strictly isothermic radiating atmosphere cannot exist.

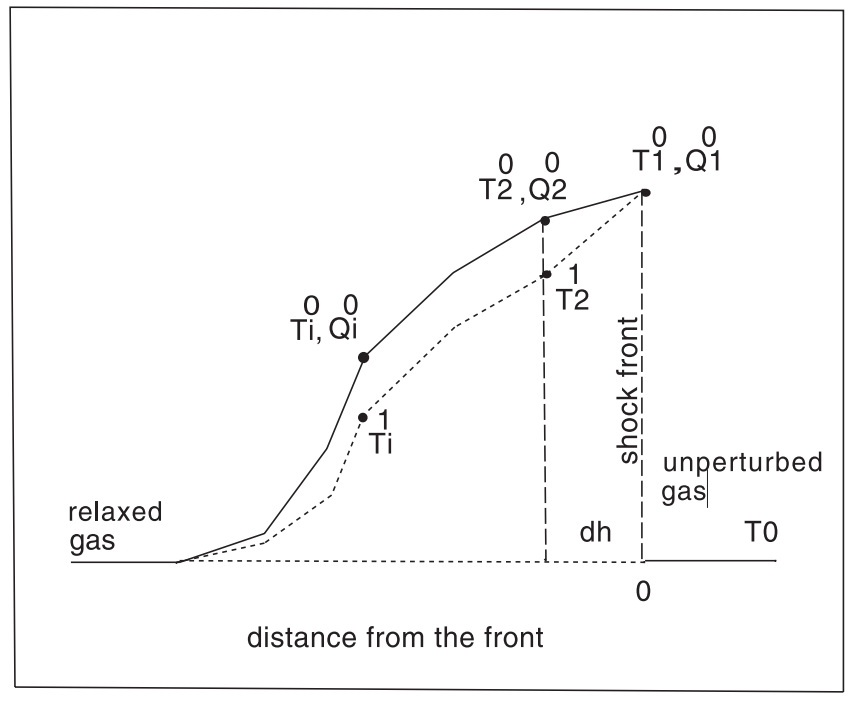

Fig. 4. Illustration of the iterative calculation of the temperature in the shock wake.

In normal atmospheres, the specific intensity is strongly anisotropic and $J_{v}$ depends mostly on the conditions at $\tau_{v} \approx 1$, so that in a static atmosphere we have $J_{v} \approx B_{v}\left(T_{\text {eff }}\right) / 2$. To satisfy the condition of the radiative equilibrium, we must put at each point $J_{v}=S_{v}$, or $S_{v} \approx B_{v}\left(T_{\text {eff }}\right) / 2$. These relations are compatible with the LTE approximation $S_{v}=B_{v}(T)$ only if $T$ is not constant within the atmosphere.

In the case without shock, $T=$ const., so in a static atmosphere and under LTE, we must have in the same time $J_{v} \approx B_{v}(T) / 2$ and $J_{v}=B_{v}(T)$, which is impossible. If we wish to keep the condition of the radiative equilibrium, which is most important, we must adopt $S_{v} \approx B_{v}(T) / 2$ at each point of our isothermic atmosphere. Thus, we have for the continuum cooling rate

$Q_{\text {cont }}=\frac{4 \pi}{\rho}\left(\bar{k}_{\mathrm{P}}\left(T_{0}\right) \frac{\sigma T_{0}^{4}}{2 \pi}-\bar{k}_{\mathrm{P}}(T) \frac{\sigma T^{4}}{2 \pi}\right)$,

where $\bar{k}_{\mathrm{P}}(T)$ is the Planck mean opacity for the local temperature $T$. Because the radiative wake is extremely opaque to the Lyman continuum, Lyman photons remain blocked and cannot contribute to the cooling (i.e. $J_{v} \approx S_{v}$ in Eq. (30)). So we did not include the Lyman continuum in $\bar{k}_{\mathrm{P}}(T)$. For practical reasons, we performed the integration of $\bar{k}_{\mathrm{P}}(T)$ from $v_{1}=10^{10} \mathrm{~Hz}$ to $v_{2}=1.11 \times 10^{16} \mathrm{~Hz}$.

The method of calculation of the temperature in the wake is illustrated in Fig. 4.

\subsection{Numerical procedure}

The whole atmospheric region is subdivided into 150 logarithmically distributed (with respect to the distance from the shock front) spherical zones, with 50 of them located in the preshock (unperturbed) region.

The radius of the front was always fixed at $100 R_{\odot}$, which gives a good approximation to a plane-parallel shock.

In the preshock, we adopt a constant mass density $\rho_{0}$ and a constant temperature $T_{0}$, although we resolve the transfer 
equation in the entire atmosphere. The gas temperature at our "shock front", $T_{1}^{0}=T_{\mathrm{s}}$, is one of the input parameters of the problem and is kept constant during the iterations.

The general calculation strategy is as follows. We start with some initial temperature distribution in the shock wake, $T^{0}(z)$, where $z$ is the distance from the front. The choice of the form of this distribution does not affect the final solution. Applying the two first Rankine-Hugoniot relations (Eqs. (4) and (5)), as mentioned above, we calculate the density and the velocity of the gas at each point of the wake. Then, resolving the transfer equations for all lines (Eq. (10)), we obtain the first approximations for the cooling rates $Q_{\text {line }}(z)$ (Eq. (29)) and $Q_{\text {cont }(z)}$ (Eq. (31)) as functions of the distance $z$. Using an explicit numerical scheme to resolve the Eq. (2), we get a new $T^{1}(z)$, which immediately gives Eqs. (4) and (5) new $\rho(z)$ and new velocities. After that, new $Q(z)$ are calculated from the transfer equation, and the iterative procedure is repeated until it converges to the exact solution.

However, this method cannot be applied directly because the $(i+1)$ th approximation $\left(T^{i+1}(z)\right)$ is too sensitive to the variations of the previous one $\left(T^{i}(z)\right)$, and the whole procedure becomes unstable. Convergency can be achieved by restricting the variations of $T^{i+1}(z)-T^{i}(z)$ in the course of iterations. For this, we use a simple linear local relation to correct the $(i+1)$ th approximation: $T_{\text {corr }}^{i+1}=f \cdot T^{i+1}+(1-f) \cdot T^{i}$, where the constant factor $f$ decreases as the convergency accelerates.

Even after this correction the iterative procedure still may remain unstable. For example, as iterations start converging, in the middle of the wake some spatial oscillations of the solution occur, growing with the number of iterations. To avoid this instability, after each iteration the solution (corrected) is regularised by a simple averaging procedure $\left(T_{j}\right)_{\text {reg }}=\left(T_{j-1}+\right.$ $\left.T_{j+1}\right) / 2$, where $T_{j}$ is the temperature at the $j$ th node.

The calculation over all iron lines is organized as follows. Tables of the atomic constants for the Fe I and Fe II lines (obtained from Kurucz' line lists) are first transformed into a single 3D table, where the dimensions are: the frequency $v_{L}, \log \left\langle g f_{L}\right\rangle$, and $E_{l}$ (the excitation energy of the lower level). Each "cell" of the table contains the number of the lines, whose parameters correspond to the "cell" coordinates. The frequency runs from $1000 \AA$ to $20000 \AA$ ( 10 uneven bins, $\log \langle g f\rangle$ from -2.5 to 1.5 ( 5 even bins) and $E_{l}$ from 0 to $9.28 \mathrm{eV}$ ( 9 uneven bins). The frequency interval between boxes are small enough to neglect the variation of the continuum opacity within one box. The cooling rate, found for each averaged line (one cell) is then multiplied by the corresponding number of lines, and then a summation over all cells is performed. Finally, we get the total cooling rate with continuum contribution, and a new approximation of the temperature distribution can be obtained. Iterations converge rapidly and monotonically to the solution which always lies between the input $T^{i}(z)$ and output $T^{i+1}(z)$ temperatures, as seen in the Fig. 5. The diagram represents a typical variation of the temperature profile during iterations. In the shown case, the preshock density is $2 \times 10^{-12} \mathrm{~g} / \mathrm{cm}^{3}, v_{0}=50 \mathrm{~km} \mathrm{~s}^{-1}$, $T_{0}=5000 \mathrm{~K}, T_{\mathrm{s}}$ at the "front" is $10000 \mathrm{~K}$ and the Fe abundance corresponds to the Population I.

The precision is controlled in two ways. Firstly, at each iteration we check the maximum of the absolute difference

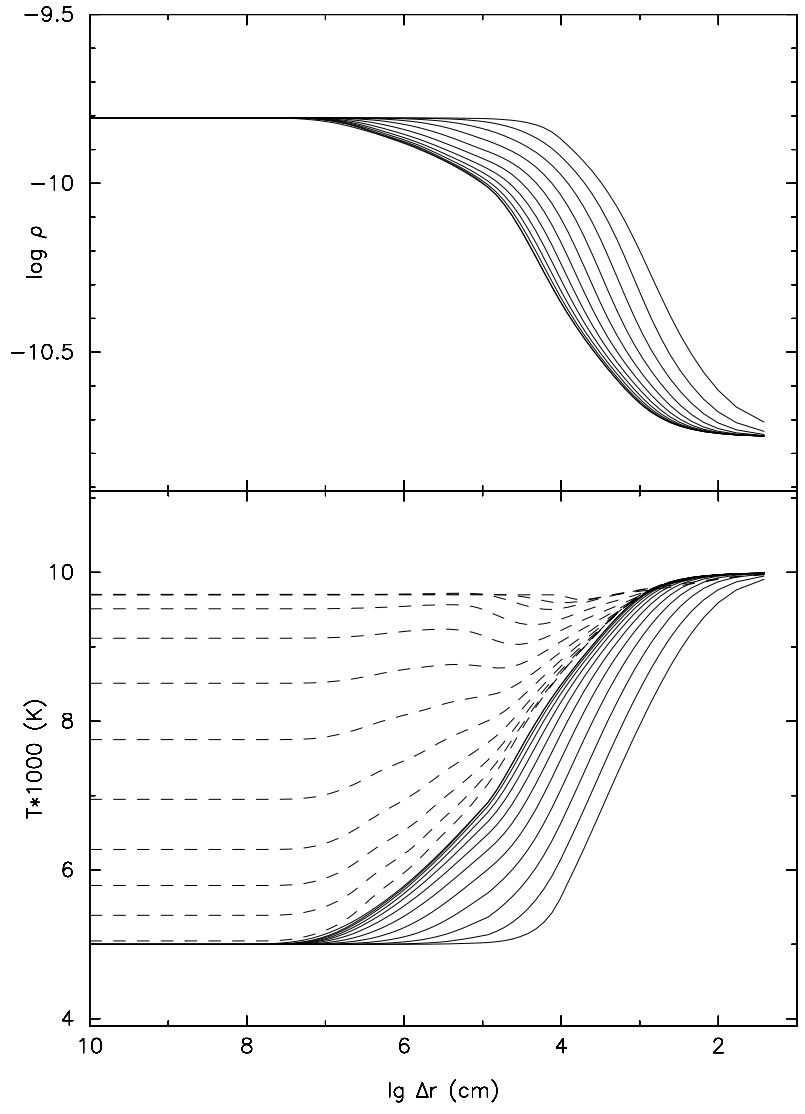

Fig. 5. Illustration of the convergency of iterations. Upper diagram shows the density profiles, lower diagram - the temperature profiles. Solid lines, converging from the right to the left in the course of iterations, show the input temperature distributions, while the dashed curves, evolving from up to the bottom, are the output distributions. The pairs of these curves monotonically approach the solution (seen as a thickening of the curves in the middle).

between the "input" $T^{i}(z)$ and "output" $T^{i+1}(z)$ obtained without the restriction procedure. Usually, this difference monotonically decreases with the number of iterations, and after about 20 iterations diminishes to $50-100 \mathrm{~K}$. Secondly, we check the current correction in $T^{i}(z)$ after the restriction procedure (the "inner" precision). This correction is much less than $T^{i}(z)-T^{i+1}(z)$, so that after 10-20 iterations normally we have $\Delta T^{i}(z) / T^{i}(z)$ less than $10^{-3}$. At this value we stopped the iterations to save the CPU time. We estimate the absolute accuracy of our calculations of $T(z)$ as less than $100 \mathrm{~K}$. However, in some rare cases the solution in the middle of the wake can stabilize, so the difference between the "input" and "output" temperatures can increase up to $300 \mathrm{~K}$, which corresponds to an error in the wake width of about 2-5 per cent.

\section{General results - cooling in stellar atmospheres}

We tried different input parameters of the model to represent the various condition in pulsating stellar atmospheres. We also tried the chemical abundance for both Populations I and II. Below we discuss the systematic changes of the temperature 


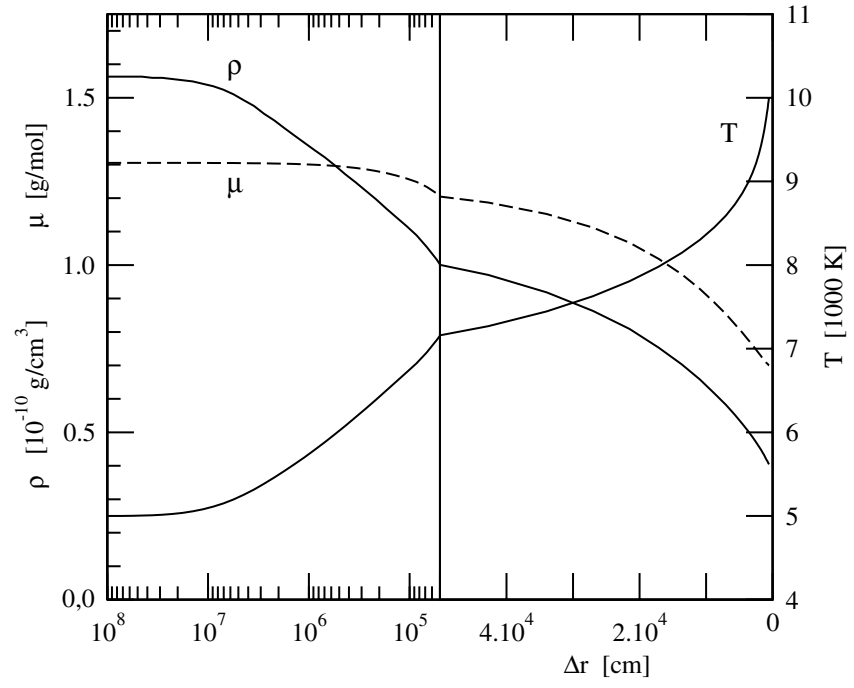

Fig. 6. Temperature, density and mean molecular weight profiles in the radiative wake for a shock with preshock parameters $T_{0}=5000 \mathrm{~K}$, $\rho_{0}=2 \times 10^{-12} \mathrm{~g} \mathrm{~cm}^{-3}$, and $v_{0}=50 \mathrm{~km} \mathrm{~s}^{-1}$ (solar abundance), as a function of the distance from the shock front. Note that the distance scale changes from linear to logarithmic.

profile in the shock wake with the variation of these input parameters, and the comparison of the cooling effects due to iron lines and hydrogen continuum.

\subsection{A typical case}

Before going to a comparison of different shocks we analyse with some depth a given case. We concentrate on a shock with parameters $T_{0}=5000 \mathrm{~K}, \rho_{0}=2 \times 10^{-12} \mathrm{~g} \mathrm{~cm}^{-3}, v_{0}=50 \mathrm{~km} \mathrm{~s}^{-1}$ and $T_{\mathrm{s}}=10000 \mathrm{~K}$. The temperature, density and mean molecular weight profiles in the wake obtained after convergency are plotted in Fig. 6. A linear distance scale is used close to the front so as to emphasize their rapid variations in that region. The evolution then becomes much smoother and is plotted on a logarithmic scale. The temperature needs around $3 \times 10^{4} \mathrm{~cm}$ to lose $2500 \mathrm{~K}$ from $10000 \mathrm{~K}$ to $7500 \mathrm{~K}$, then a distance more than a thousand times larger to lose the same amount and relax to $5000 \mathrm{~K}$. Trends for the density are similar because $\rho$ is related to $T$ through Eq. (6). It increases however by a factor of 3.8 while the temperature is divided by 2 , as the mean molecular weight is at the same time multiplied by a factor of 1.9 due to hydrogen recombination (see Fig. 6). The latter process takes place close to the front where most of the radiated gas energy is deducted from the ionization pool.

We compare in Fig. 7 the behaviour of strong and weak lines. Different quantities are plotted in each panel for all the model lines of Fe II that fall in the wavelength bin around $2500 \AA$. Four oscillator strentgh values are considered $(\log g f=0.5$ to -2.5$)$, and six lower level excitation energies (from $0 . \mathrm{eV}$ to $5.57 \mathrm{eV}$ ) that makes a total of 24 model lines. These lines represent a total of some two thousands real lines. Three peculiar lines are shown in the plots: the strongest one and two weaker ones due to either a lower $g f$ value or a higher excitation energy $E_{l}$.

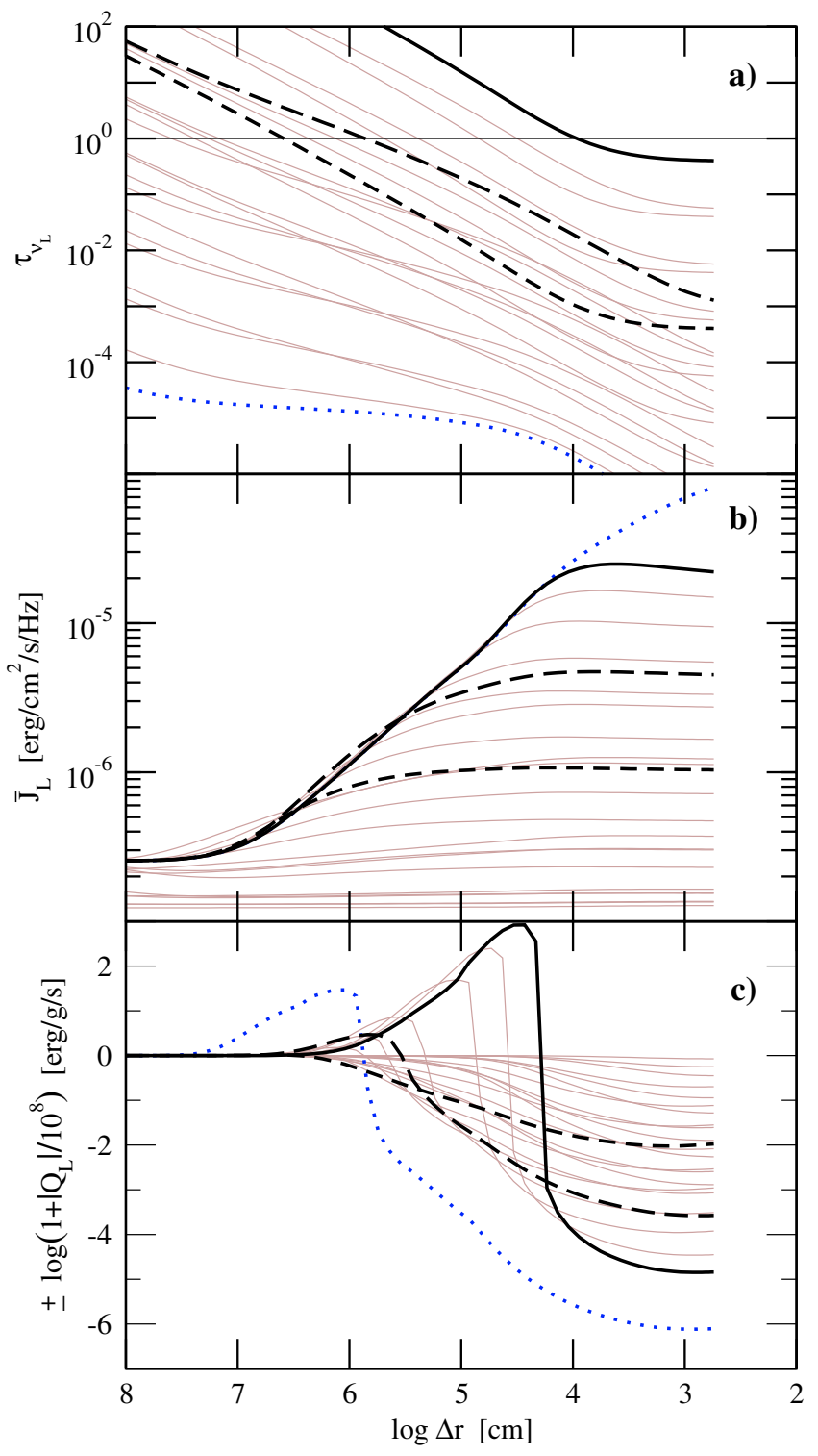

Fig. 7. Behaviour as a function of the distance from the shock front of some iron line quantities, for the same shock as in Fig. 6. All model lines of Fe II that are in the wavelength being centered at $2500 \AA$ are plotted. They differ in their oscillator strengths and/or lower level excitation energies. a) optical depth $\tau_{v_{L}}$ (in the line center); b) scattering integral $\bar{J}_{L} ;$ c) contribution $Q_{\text {lines, } L}$ to the cooling rate (through one line; note the vertical scale allowing logarithmic plot of both negative and positive values). Some peculiar lines are emphasized in the three subpanels through heavy curves: (solid) $\log g f=0.5$, $E_{l}=0 . \mathrm{eV}$; (long dashed) $\log g f=0.5, E_{l}=2.9 \mathrm{eV}$; (short dashed) $\log g f=-2.5, E_{l}=0 . \mathrm{eV}$. The heavy dotted curve has different meanings: a) continuum optical depth (at this wavelength); b) source function $S_{L}=B_{v_{L}}(T)$; c) total cooling rate due to all these lines (taking account of the effective number of lines in each cell).

As seen in Fig. 7a the shock front is thin for all the lines. The optical depth reaches unity for the strongest line at a distance $\sim 10^{4} \mathrm{~cm}$ from the front and weakest ones remain transparent even far in the wake, as for the continuum. The solution of the radiative transfer leads for each line to the scattering integral $\overline{J_{L}}$, which is depicted in Fig. $7 \mathrm{~b}$ and compared to the Planck source function $S_{L}=B_{v_{L}}(T)$. Close to the front the value of the 


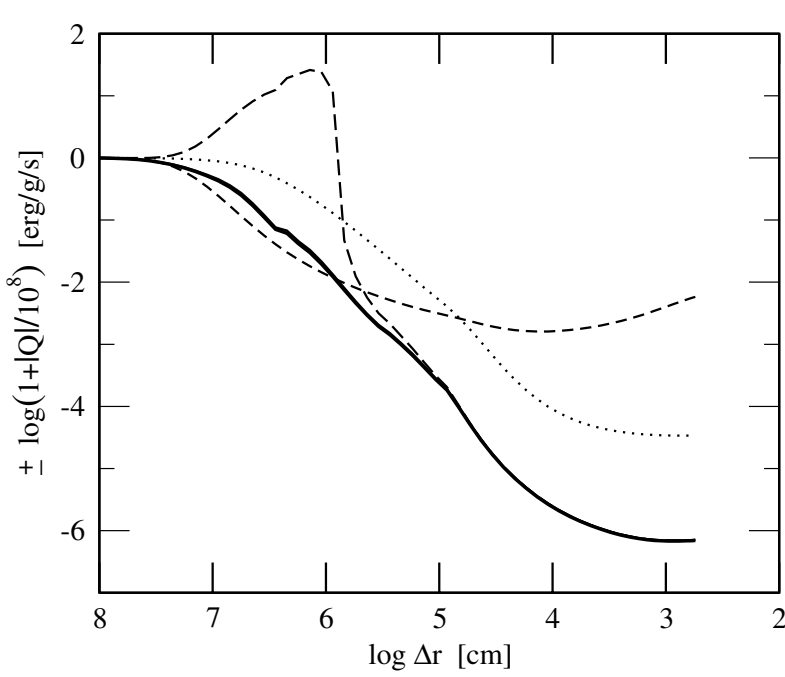

Fig. 8. Cooling rates as a function of the distance from the shock front for the same shock as in Fig. 6. Dotted line: continuum cooling rate; short-dashed line: Fe I cooling rate; long-dashed line: Fe II cooling rate; solid heavy line: total rate.

ratio $\overline{J_{L}} / S_{L}$ is between 0 and 0.27 , in agreement with Fig. 7 of FMG1 and the related analysis. Consequently in the second line of Eq. (29) one has $\left(\overline{J_{L}} / S_{L}-1\right) \simeq-1$. The cooling capacity of a line is merely determined by the product of its oscillator strength times its lower level population. This may be verified in Fig. 6c. The short-dashed line cools a thousand times less than the strongest one because of the oscillator strengths ratio, while the long-dashed one cools a factor 0.04 less because of the $\exp \left(-E_{l} / k T\right)$ factor.

The situation becomes more interesting farther in the wake. As is apparent from a comparison of Fig. 7a and b, the ratio $\overline{J_{L}} / S_{L} \simeq 1$ when lines become opaque $\left(\tau_{L} \gtrsim 1\right)$. Cooling then transforms into heating (Fig. 7c). The strongest lines lead to a greater heating than (not too) weak ones. While the factor $\left(\overline{J_{L}} / S_{L}-1\right)$ is larger for the latter than the former (a maximum of 0.4 versus 0.04 in this case), weak lines heat farther in the wake. The lower temperatures compensate through $B_{v}(T)$ the increase of the factor. Note that when compared to Figs. 4 6 of FMG1 the maximum ratios $\overline{J_{L}} / S_{L}$ we find here are much lower. This is due to a different temperature profile. The very extended wake tail we compute here self-consistently was absent in FMG1 (see Fig. 3 in that paper).

The final cooling rate can be obtained only from a detailed calculation including the radiative transfer solution. Compensations occur between cooling/heating lines as soon as the "thin" layer behind the front is crossed. In our present case the total rate (for the lines around $2500 \AA$ ) is depicted as the dotted line in Fig. 7c. The strong lines heating between $\log \Delta r=4.2$ and 5.5 is more than balanced by the cooling of weak lines which are much more numerous. Those lines combine into a heating zone between $\log \Delta r=6$ and 7 .

In Fig. 8 the respective contributions of Fe I, Fe II and of the continuum are plotted together with the total cooling rate for the same shock. The Fe II total cooling rate is almost given by the mere contribution of the lines in the $2500 \AA$ range (see previous figure). While up to $\log \Delta r \simeq 5.5$ the neutral iron contribution is dominated by the Fe II one, it compensates the heating zone of the latter farther in the wake. Because of its low fractional abundance in all the shocks we consider in this paper, Fe I always has optically thin lines that heat far in the wake, although with a modest value. The continuum is everywhere a very weak cooler in that weak shock case, but its contribution can become more important as the shock becomes stronger as we will now see.

\subsection{Dependence on the preshock parameters}

In the following examples we adopt the shock peak temperature $T_{\mathrm{s}}=10000 \mathrm{~K}$ and the unperturbed temperature $T_{0}=5000 \mathrm{~K}$. The value of $T_{\mathrm{s}}$ will be discussed later. Such a temperature is found plausible for the beginning of the radiative relaxation zone. $T_{0}=5000 \mathrm{~K}$ is characteristic for most pulsating stars, like classical Cepheids, W Virginis or RV Tauri stars. We also tried $T_{0}=3000 \mathrm{~K}$ and $T_{0}=7000 \mathrm{~K}$, relevant for cooler Miras and hoter RR Lyrae stars, respectively. The velocities of the shock varied from $30 \mathrm{~km} \mathrm{~s}^{-1}$ to $150 \mathrm{~km} \mathrm{~s}^{-1}$, covering the velocity spectrum from Miras to RR Lyrae stars. The preshock densities were varied from $2 \times 10^{-13}$ to $2 \times 10^{10} \mathrm{~g} / \mathrm{cm}^{3}$, which is typical for atmospheres of these stars.

In Fig. 9 we present the dependence of the radial distribution of different physical values in the wake, calculated with the continuum and $\mathrm{Fe}$ lines, on the preshock density $\rho_{0}$ and gas velocity $v_{0}$ for the Population I abundance. The upper panel of each diagram shows the gas temperature as function of the distance from the front (in the sense discussed before), the middle panel represents the ratio of the cooling rates (per gram and per second) due to iron lines and the continuum. The bottom panel shows the cooling rate $Q_{\text {lines }}+Q_{\text {cont }}$.

It clearly appears that the line cooling strongly dominates the continuum cooling at smaller preshock densities, typically for $\rho<10^{-11} \mathrm{~g} / \mathrm{cm}^{3}$.

In Fig. 10 we compare the solutions with the $\rho_{0}=$ $2 \times 10^{-11} \mathrm{~g} / \mathrm{cm}^{3}$ and $v_{0}=50 \mathrm{~km} \mathrm{~s}^{-1}$ for the two stellar Populations, I and II. The effect of the underabundance of iron (Pop. II) strongly minimizes the cooling due to iron lines, so the line cooling in dense Pop. II atmospheres is not considerable. On the contrary, the continuum cooling is not sensible to the abundance variations, because it is controlled mainly by hydrogen and helium opacities.

Figure 11 shows the non-LTE effect, approximated by Eq. (16), on the solution. Rather unexpectedly, we see that as $\epsilon$ approaches $0.1, Q_{\text {line }(z)} / Q_{\operatorname{cont}(z)}$ strongly increases in the inner half of the wake, although closer to the front it slightly diminishes. As a consequence, at $\epsilon=0.1 T$ decreases faster than at $\epsilon=1$. This seems strange in view of the fact that the non-LTE effects (usually the bound-bound scattering) push the value of $\bar{J} / S$ toward unity. This result is explained in Appendix 1 by a test one line calculation, and is illustrated in Fig. A.1. 

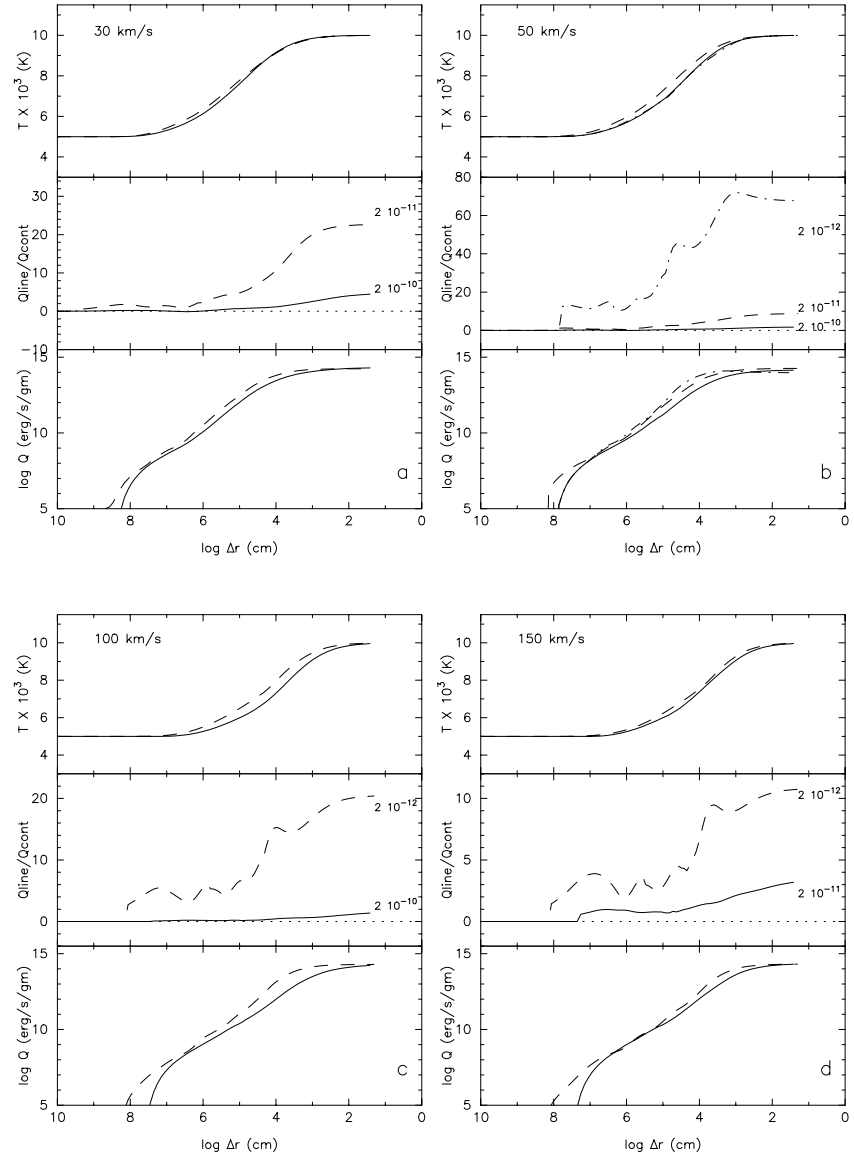

Fig. 9. Variations of different physical quantities in the shock wake with the distance from the shock front as function of the preshock density and gas velocity for Pop. I composition: a) $v_{0}=30 \mathrm{~km} \mathrm{~s}^{-1}$, solid: $\rho_{0}=2 \times 10^{-10} \mathrm{~g} / \mathrm{cm}^{3}$, dashed: $\left.\rho_{0}=2 \times 10^{-11} \mathrm{~g} / \mathrm{cm}^{3} ; \mathbf{b}\right) v_{0}=$ $50 \mathrm{~km} \mathrm{~s}^{-1}$, same legend as for a); c) $v_{0}=100 \mathrm{~km} \mathrm{~s}^{-1}$, solid: $\rho_{0}=2 \times$ $10^{-10} \mathrm{~g} / \mathrm{cm}^{3}$, dashed: $\left.\rho_{0}=2 \times 10^{-12} \mathrm{~g} / \mathrm{cm}^{3} ; \mathbf{d}\right) v_{0}=150 \mathrm{~km} \mathrm{~s}^{-1}$, solid: $\rho_{0}=2 \times 10^{-11} \mathrm{~g} / \mathrm{cm}^{3}$, dashed: $\rho_{0}=2 \cdot 10^{-12} \mathrm{~g} / \mathrm{cm}^{3}$.

\subsection{Lines vs. continuum}

Figure 12 demonstrates the effect of the iron lines on the temperature profile in the wake with respect to the continuum. The dashed curves correspond to the cooling due to continuum only, and the solid curves to the continuum and Fe-lines. The diagrams were obtained for $T_{0}=5000 \mathrm{~K}, T_{\mathrm{s}}=10000 \mathrm{~K}$, and different velocities and densities of the preshock gas. Diagrams a) - c) correspond to the Population I composition, and the diagram d) shows the Population II results.

The effect of iron lines on the wake scale is most prominent for low $\rho_{0}$ and $v_{0}$, and for Population I abundance. For $v_{0}=30-50 \mathrm{~km} \mathrm{~s}^{-1}$ and $\rho_{0}=2 \times 10^{-13}-2 \times 10^{-12} \mathrm{~g} / \mathrm{cm}^{3}$ the difference in characteristic scales of the wake with and without the Fe lines increases up to $2-2.5$ orders of magnitude. For high densities and velocities this difference diminishes, and almost disappears for $\rho_{0}=2 \times 10^{-10} \mathrm{~g} / \mathrm{cm}^{3}$ and Population II case. As can be seen from the figures, such a large variation of the line effect with the input parameters is mainly due to the variation in the continuum cooling, while the Fe line cooling seems less variable with density (but depends on $v_{0}$ ). This means that practically only dashed curves move in Fig. 12.

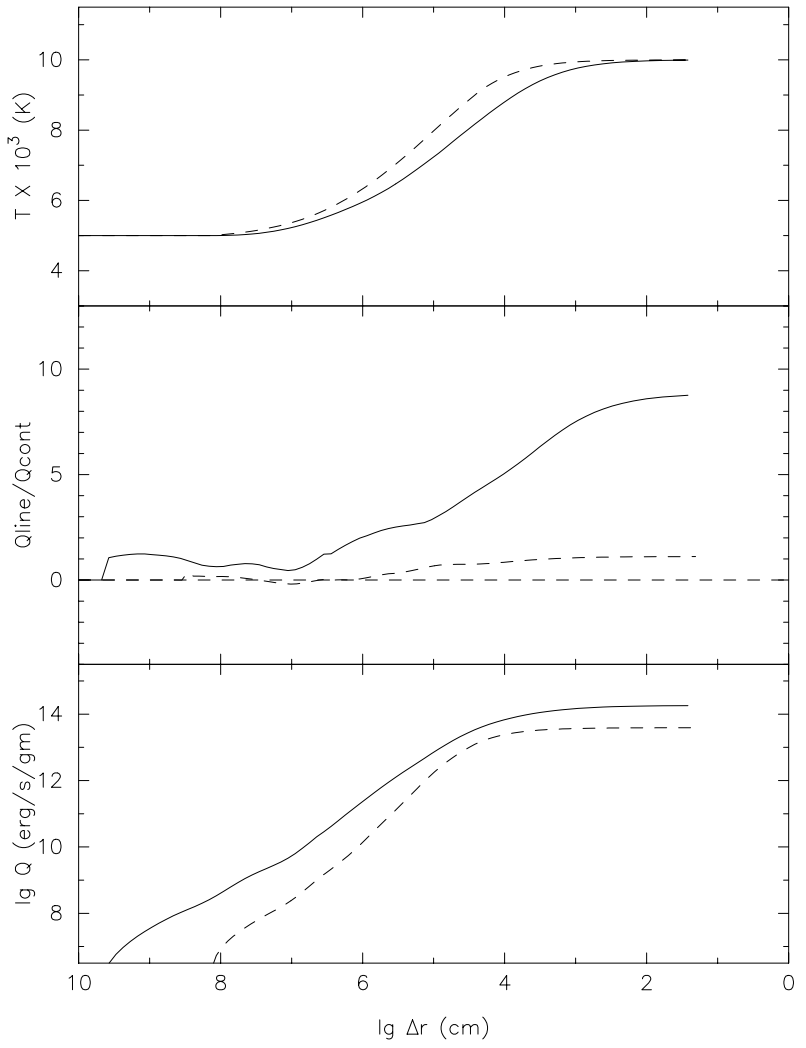

Fig. 10. Comparison of the solutions for the Pop. I (solid) and II (dashed curves). The cases correspond to $\rho_{0}=2 \times 10^{-11} \mathrm{~g} / \mathrm{cm}^{3}$ and $v_{0}=50 \mathrm{~km} \mathrm{~s}^{-1}$.

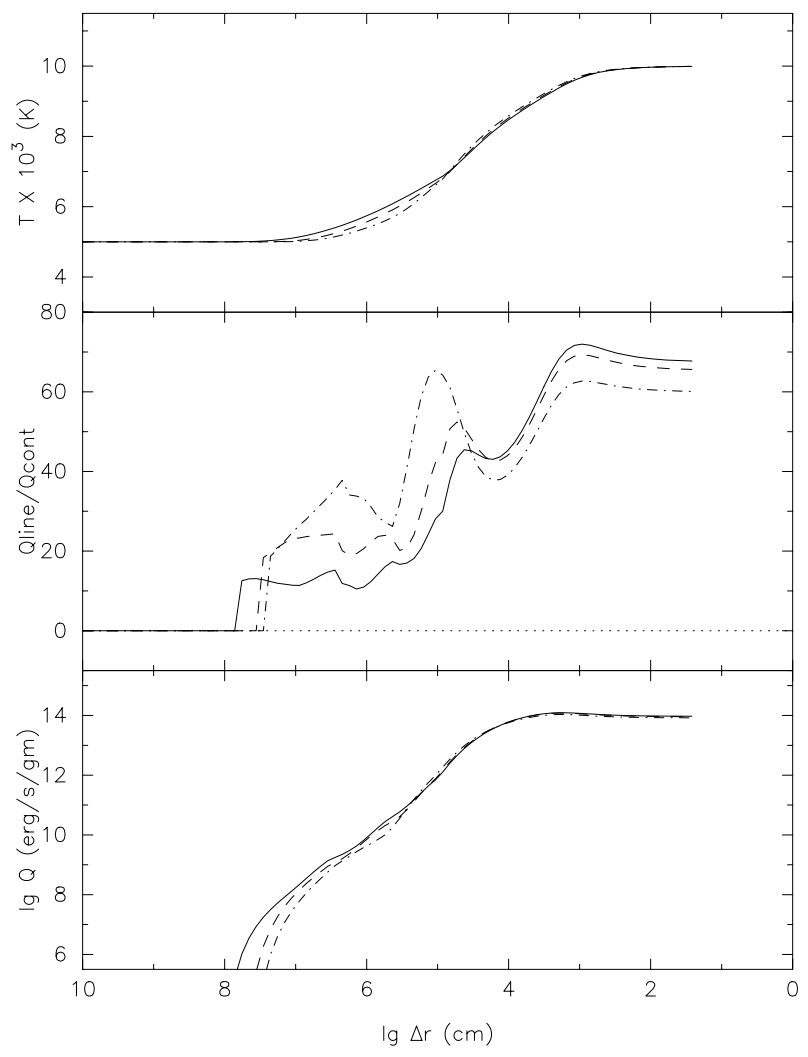

Fig. 11. Comparison of the solutions for $\epsilon=1$ (solid), $\epsilon=0.5$ (dashed) and $\epsilon=0.1$ (dot-dashed curves) for $\rho_{0}=2 \times 10^{-12} \mathrm{~g} / \mathrm{cm}^{3}$ and $v_{0}=50 \mathrm{~km} \mathrm{~s}^{-1}$. 

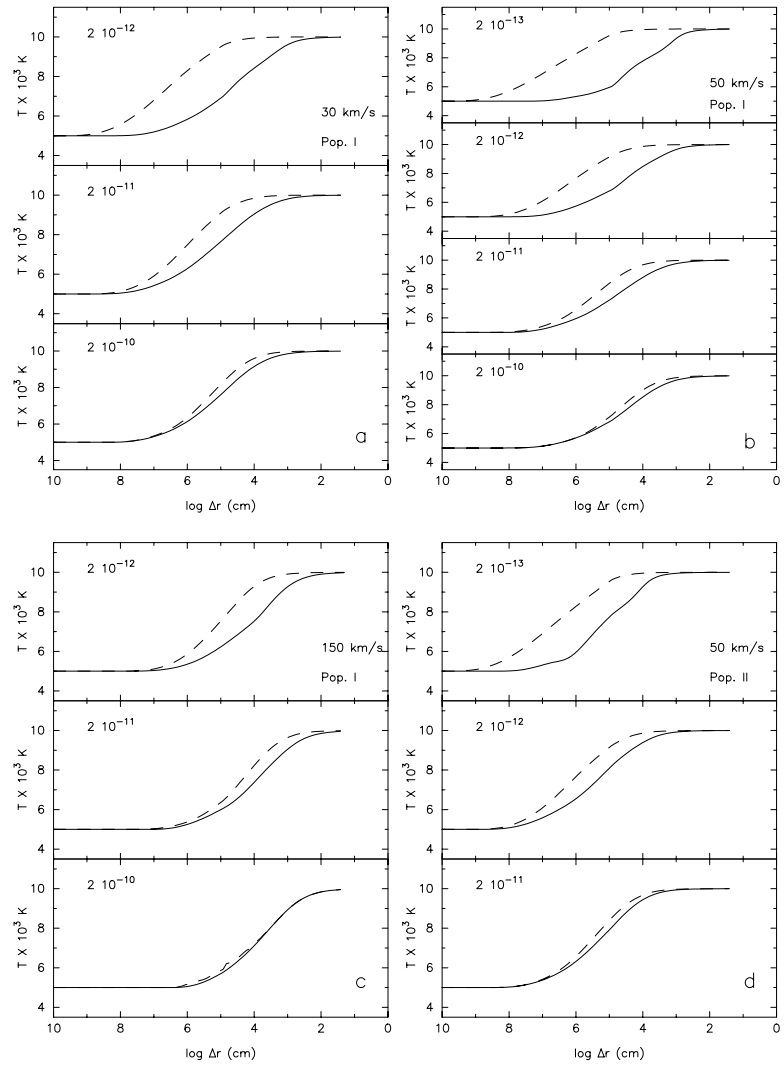

Fig. 12. Comparison of the temperature profiles in the wake, calculated with (solid curves) and without (dashed curves) Fe lines. The density in $\mathrm{g} / \mathrm{cm}^{3}$ is indicated for each pair of curves. a): $v_{0}=$ $30 \mathrm{~km} \mathrm{~s}^{-1}$, Pop. I; b): $v_{0}=50 \mathrm{~km} \mathrm{~s}^{-1}$, Pop. I; c): $v_{0}=150 \mathrm{~km} \mathrm{~s}^{-1}$, Pop. I; d): $v_{0}=50 \mathrm{~km} \mathrm{~s}^{-1}$, Pop. II.

\subsection{Remarks on the choice of $T_{\mathrm{s}}$}

Now we wish to discuss the important question: is our choice of $T_{\mathrm{s}}$ right, and if not, what temperature should be taken in the beginning of the wake? Indeed, immediately after the viscous front, two temperatures co-exist - one for electrons and one for heavy particles ( the last temperature can reach $10^{5} \mathrm{~K}$ and more). As known, at the point of the thermalization $\left(T_{\mathrm{e}}=T_{a}=\right.$ $\left.T_{i}\right), T$ is strongly diminished, but its value is not easy to estimate. We can only expect (see e.g., Narita 1973, or Fadeev $\&$ Gillet 2000, 2001) that, depending on physical conditions, this temperature is about $20-25 \times 10^{3} \mathrm{~K}$ and the distance of this point from the viscous front must be less than about one meter. The physical conditions here are still far from LTE. For example, the radiation in the Balmer and, which is more critical, Lyman continua is strongly anisotropic. However, at $T \approx 10^{4} \mathrm{~K}$ the Lyman continuous radiation is already blocked, and the Balmer fluxes in both directions become much closer. Thus, we may hope that at such temperatures our model becomes plausible.

Unfortunately, we have no means to prove directly our main hypothesis, i.e. that the region between our "front" and the viscous shock front is effectively transparent for all iron lines. In our numerical analysis, we used $T_{\mathrm{s}}=10000 \mathrm{~K}$ without explanation. Now we wish to test this choice using the following reasoning. If the region with $T>10000 \mathrm{~K}$ is effectively

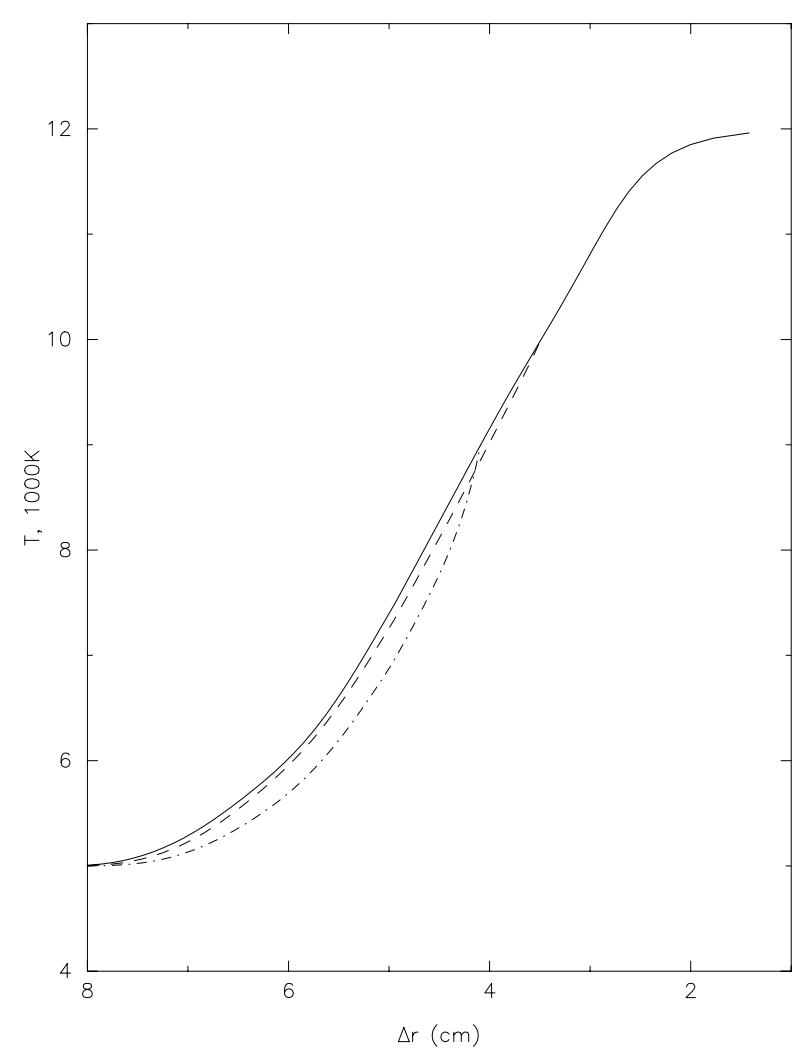

Fig. 13. An example of the comparison of solutions with different values of $T_{\mathrm{s}}$ in order to find the "critical" temperature at the "shock front" (see text). Solid: $T_{\mathrm{s}}=12000 \mathrm{~K}$; dashed: $T_{\mathrm{s}}=10000 \mathrm{~K}$; dot-dashed: $T_{\mathrm{s}}=9000 \mathrm{~K}$.

transparent to the iron line radiation, then this zone does not contribute much to the temperature decrease due to the radiative cooling. In this case, the solutions obtained with $T_{\mathrm{s}}=$ $10000 \mathrm{~K}$ and, say, $T_{\mathrm{s}}=12000 \mathrm{~K}$ must be close. Equally, if the region with $T>9000 \mathrm{~K}$ is still transparent for the Fe lines, there must not be much difference between the solutions with $T_{\mathrm{s}}=9000 \mathrm{~K}$ and $T_{\mathrm{s}}=10000 \mathrm{~K}$, etc. In this comparison, we should shift the scales of different solutions in order to fit the $T_{0}$ of one solution to the temperature curve of the another. By this procedure, we can estimate the "critical" $T_{\mathrm{cr}}$, so that all $T_{\mathrm{s}}>T_{\text {cr }}$ satisfy our condition of the transparency in the lines.

We performed such a comparison, trying 3 values of $T_{\mathrm{s}}$ : 9000,10000 and $12000 \mathrm{~K}$ for 9 different sets of input parameters of our shock model. An example of such a test is presented in the Fig. 13 for $v_{0}=50 \mathrm{~km} \mathrm{~s}^{-1}, \rho_{0}=2 \times 10^{-11} \mathrm{~g} / \mathrm{cm}^{3}$ and Pop. I. For instance, from this figure one can deduce $T_{\mathrm{cr}} \approx 10000 \mathrm{~K}$.

In all the other 8 studied cases we found $T_{\text {cr }}$ being around $10000 \mathrm{~K}$, with only 2 exceptions: when, after additional detailed calculations, $T_{\mathrm{cr}}$ was found at $11000-12000 \mathrm{~K}$. So we believe that by this our initial choice of $T_{\mathrm{s}}=10000 \mathrm{~K}$ is justified.

\section{Line cooling in different types of pulsating stars}

It is interesting to apply our results to different types of pulsating stars. We demonstrated above that the line cooling is 


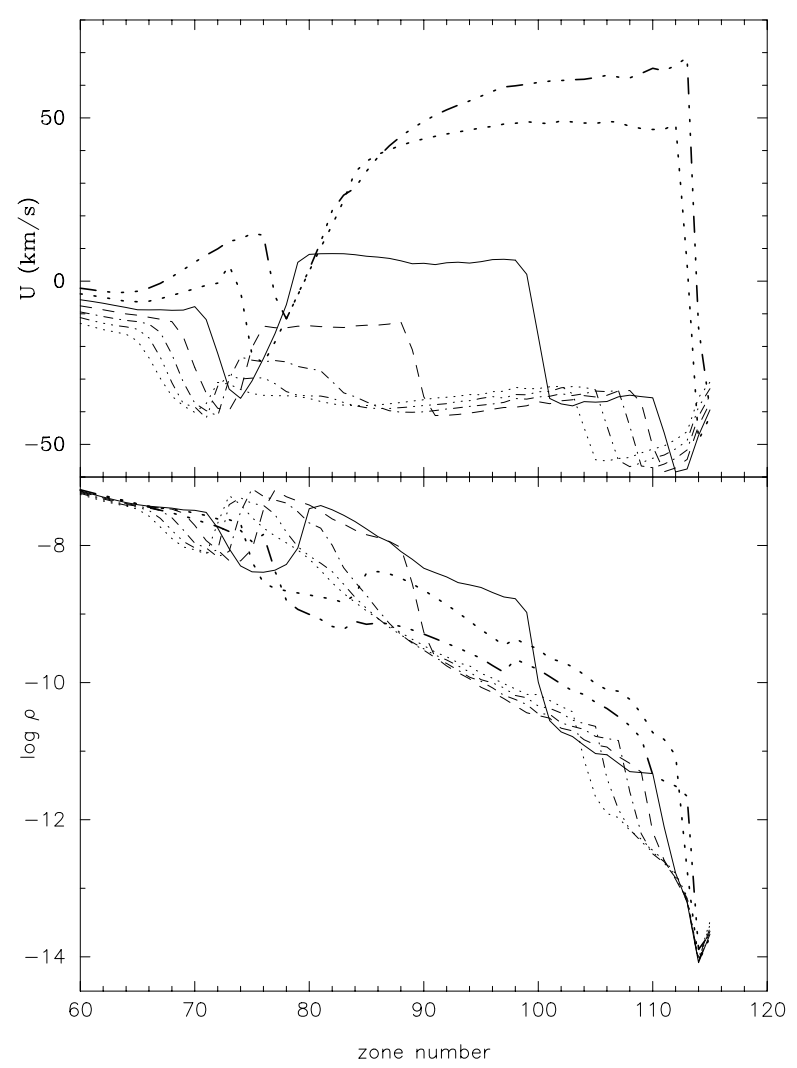

Fig. 14. Typical temporal evolution of the radial velocity and mass density profiles in an RR Lyrae model during the phase of a shock propagation according to a non-linear model. The shock moves from left to right.

very strong with respect to continuum only at relatively low preshock densities $\left(\rho<2 \times 10^{-11} \mathrm{~g} / \mathrm{cm}^{3}\right)$. To examine the possible effect of the line cooling in pusating stellar atmospheres, we estimated typical densities that can occur before a shock in stars of different types. For this, we used the hydrodynamical models, calculated in our previous works (Fokin 1992, 1994; Fokin et al. 1996), related to the RR Lyr, W Vir, RV Tauri and classical Cepheid variables.

In Figs. 14 and 15 we present the time evolution of the radial velocity and mass density profiles in the pulsating atmospheres of RR Lyrae and RV Tau models during the phases of shock generation and propagation. These two models represent two extreme cases of stars with low (RR Lyr, classical Cepheids) and high (W Virginis, RV Tauri, Miras) radius-tomass ratio. The horizontal coordinate is the mass zone number, counted from the inner boundary of the model. The shock (the velocity and density jump) displaces from left to right (i.e. to the surface).

These diagrams demonstrate the generally known fact that stars with high $R / M$ ratio (low gravity) have atmospheres with low density gradients, while the density in the atmospheres with low $R / M$ (high gravity) quickly decreases towards the surface. In the last case, the preshock density can easily be less than $10^{-12} \mathrm{~g} / \mathrm{cm}^{3}$, so, according to our present results, in these stars the cooling effect due to the Fe lines must be important. This is the case of classical Cepheids and RR Lyrae stars. We anticipate that the iron cooling must be especially

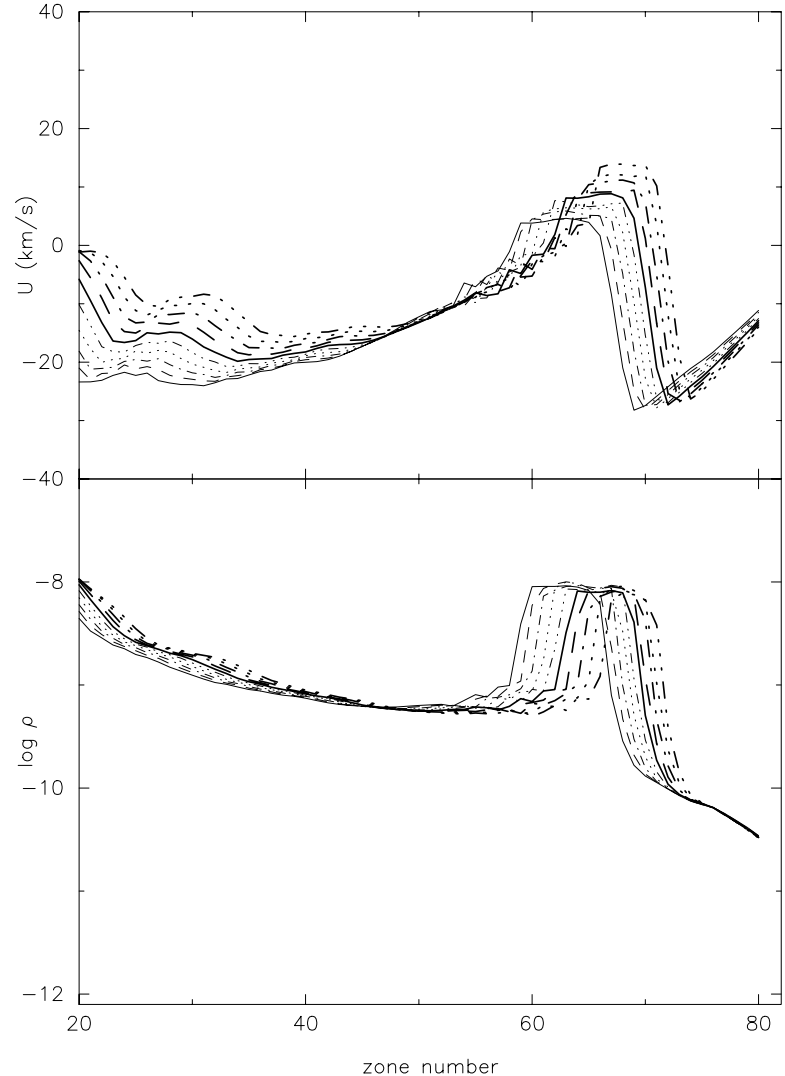

Fig. 15. Same as in Fig. 10 for an RV Tauri model.

important for the classical Cepheids, since, in addition, they have Population I composition. As a result, in these stars the hot wake should be considerably reduced, so that the expected line emission, including $\mathrm{H} \alpha$, would be less prominent than in stars having high $R / M$ ratio. In this context, it is interesting to note that in RR Lyr pulsating stars, despite the very high shock amplitude, up to $200 \mathrm{~km} \mathrm{~s}^{-1}$, the emission in $\mathrm{H}$ lines is very rare. In classical Cepheids the line emission is observed only in a few stars with extremely long periods (higher $R / M$ ratio?). On the contrary, in the W Virginis, RV Tauri and Mira stars, strong Balmer and metallic line emissions are seen almost permanently. We speculate that such a spectroscopic difference between these two stellar types can have its origin in different efficiency of the iron cooling.

\section{Conclusions}

In the course of detailed calculations of the hot radiative shock wake structure in stellar atmospheres with the effect of iron line cooling we found that:

- the iron cooling can be very effective with respect to the hydrogen continuum if only the preshock density is relatively low. For Population I composition and for $\rho$ about or less than $10^{-12} \mathrm{~g} / \mathrm{cm}^{3}$, the scale of the wake diminishes by $2-2.5$ orders of magnitude due to the iron cooling. For $\rho$ about $10^{-10} \mathrm{~g} / \mathrm{cm}^{3}$, the relative contribution of the iron cooling considerably decreases, and for the Population II composition becomes almost negligible; 
- the cooling due to the Fe lines is found less affected by the model parameters than the continuum cooling, so the variation in the lines/continuum difference is mainly due to the changes in the continuum cooling rate;

- the main parameter of our model - the starting temperature at our so-called shock front, which we adopted as $10^{4} \mathrm{~K}-$ seems plausible in view that in the thin structure of the real shock, ignored in our shock model, the iron cooling can be neglected;

- the iron cooling must be most effective in variable stars with low $R / M$ ratio, like RR Lyrae or classical Cepheids, having very low atmospheric densities. On the contrary, in stars with high $R / M$, like RV Tauri, W Vir or Miras, the iron contribution to cooling is considerably reduced;

- a simplified analysis of the possible non-LTE effect on the iron line cooling shows that this effect can reduce the heating in the inner wake zone (see FMG1). As a result, without LTE the wake becomes slightly narrower.

Despite that our model allowed a self-consistent evaluation of the structure of the shock wake, several shortcomings still remain:

- although we expect that the LTE approximation gives realistic results (see Woitke \& Sedlmayr 1999), a more sophisticated study of this important problem should be performed in the future;

- it is also important to evaluate the correct continuum cooling rate, and the non-LTE effect on it. From this point of view our present approximation of the continuum cooling rate is not self-consistent;

- we did not include in our analysis the lines of other elements, so their effect should be evaluated in the future;

- we did not include in our analysis the dynamical blending of the lines in the preshock and postshock zones. The postshock velocity normally varies by less than $1-3 \mathrm{~km} \mathrm{~s}^{-1}$ within the wake, which corresponds to the Doppler shift about $0.02 \AA$ for the iron line packets. Consequently, the blending in this zone seems not very important. In the preshock zone, the gas velocity along the line of sight, depending on $\mu$, varies significantly.

We plan to further improve our shock wake models in subsequent papers.

Acknowledgements. The work of A.B.F. has been done in part under the NATO grant in 2002. Some of the present computations were carried out using the computer centre of the Observatoire de HauteProvence. A.B.F. is grateful to the staff of the OHP for the kind help.

\section{Appendix A: Non-LTE effects on the line cooling}

As seen in Fig. A.1, the non-LTE effect mostly decreases the heating in the inner part of the wake (discussed in FMG1), while the cooling is less affected and remains strong. The heating due to the Fe lines is smaller at $\epsilon=0.1$, so the total cooling in this non-LTE case is more effective. We note that, strictly speaking, this is not a more effective cooling but a weaker heating.

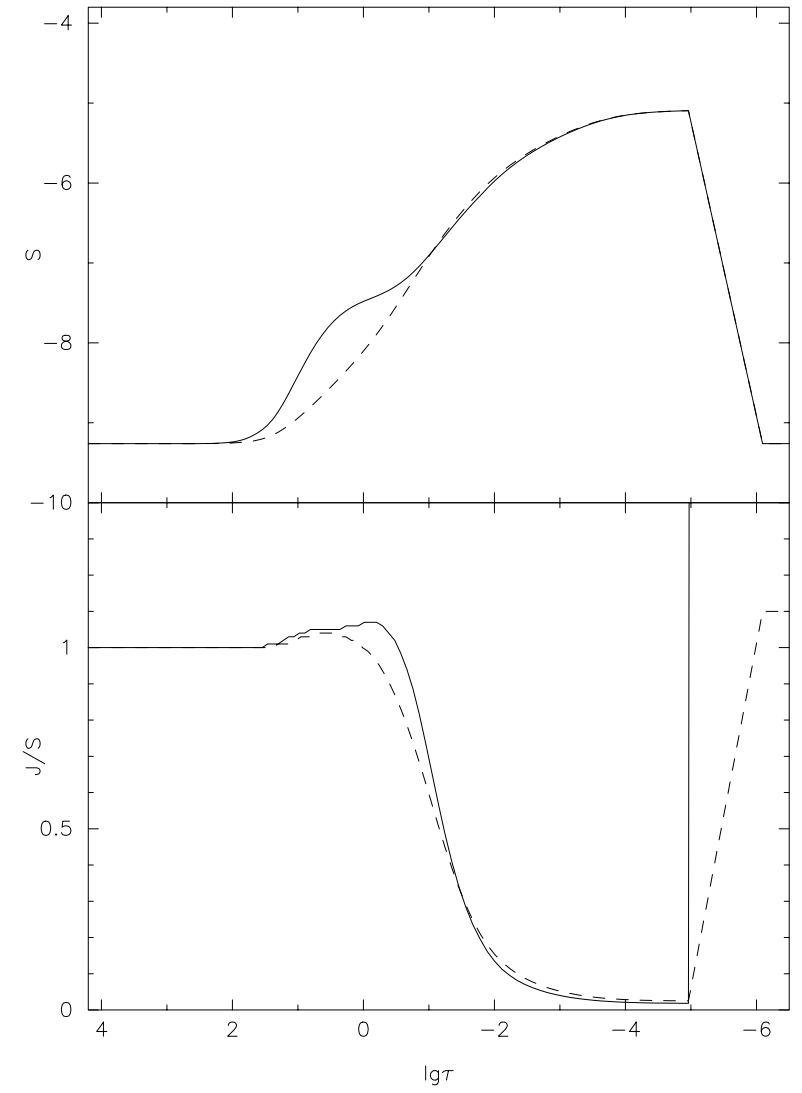

Fig. A.1. Solutions for $\epsilon=1.0$ (solid lines) and 0.1 (dashed lines) for one FeII line. Both solutions are obtained with $Q_{\text {line }}$ artificially increased by the same numerical factor. Upper diagram: the line source function as function of the static optical depth in the line center. Lower diagram: the $\bar{J} / S$ ratio, characterising the cooling/heating rate. The model corresponds to $\rho_{0}=2 \times 10^{-12} \mathrm{~g} / \mathrm{cm}^{3}, v_{0}=50 \mathrm{~km} \mathrm{~s}^{-1}$ and Pop. I.

The non-LTE effect increases as the shock displaces to the optically thick region. However, most of the Fe lines analysed here are weak, so the non-LTE effect is perceptible only in the inner part of the wake.

We remind the reader that our main calculations are performed using $\epsilon=1$.

\section{References}

Buchholz, B., Hauschildt, P. H., Rammacher, W., \& Ulmschneider, P. 1994, A\&A, 285, 987

Castor, J. I. 1972, ApJ, 178, 779

Cox, D. P., \& Tucker, W. H. 1969, ApJ, 157, 1157

Fadeyev, Yu. A., \& Gillet, D. 2000, A\&A, 354, 349

Fadeyev, Yu. A., \& Gillet, D. 2001, A\&A, 368, 901

Fokin, A. B. 1992, MNRAS, 256, 26

Fokin, A. B. 1994, A\&A, 292, 133

Fokin, A. B., Gillet, D., \& Breitfellner, M. G. 1996, A\&A, 307, 503

Fokin, A. B., Massacrier, G., \& Gillet, D. 2000, A\&A, 355, 668 (FMG1)

Hummer, D. G., \& Rybicki, G. B. 1971, MNRAS, 152, 1

Irwin, A.W. 1981, ApJS, 45, 621

Mihalas, D. 1978, Stellar atmospheres, 2nd ed. (San Francisco: W.H. Freeman and Co)

Narita, S. 1973, Progr. Theor. Phys., 49, 1911

Woitke, P., \& Sedlmayr, E. 1999, A\&A, 347, 617 